Baltic Astronomy, vol.2, 435, 1993.

\title{
PROGRESS REPORT ON THE ANALYSIS OF THE WET CAMPAIGN ON RXJ $2117+3412$
}

\author{
G.Vauclair
}

Laboratoire d'Astrophysique de Toulouse, 14 Avenue Edouard Belin, 31400 Toulouse, France.

Received August 1, 1993.

Key words: stars: white dwarfs - stars: individual: RXJ 2117+3412

\section{Abstract}

RXJ $2117+3412$ was discovered to be a bright PG 1159 type white dwarf during the ROSAT survey (see the communication by K. Werner at the White Dwarf workshop in Leicester, July 1992). In the month following the discovery announcement, rapid photometry was performed. RXJ $2117+3412$ revealed to be a new pulsating PG 1159 type star of low amplitude and with a rich spectrum, opening the opportunity to study in greater detail one more pulsator of this type, after the PG 1159-035 itself.

As a WET campaign was planned for September 1992, this exciting object was added at the last minute to the target list as a third priority object. Preliminary analysis of the results was presented at the workshop. The longitude coverage is not very good (about 30 per cent) but nevertheless the power spectra are very rich with more than 80 significant frequencies. The dominant mode has shifted to a long period of $2000 \mathrm{~s}$, compared to the August 1992 observations.

The analysis of the September 1992 WET campaign is still underway. New observations of this star are planned in September 1993 and another WET campaign on RXJ $2117+3412$ as the first priority target is planned for the 2nd semester of 1994 . 